\title{
DETERMINATION OF MAGNITUDE AND DIRECTION OF LAND USE/ LAND COVER CHANGES IN TERKOS WATER BASIN, ISTANBUL
}

\author{
F. Bektas Balcik ${ }^{\mathrm{a}, *}$, C. Goksel ${ }^{\mathrm{a}}$ \\ a ITU, Civil Engineering Faculty, 34469, Maslak Istanbul, Turkey - (bektasfi, goksel)@itu.edu.tr
}

Commission VII, WG VII/5

KEY WORDS: Land Use, Land Cover, Environment, Change Detection, SPOT, Analysis

\begin{abstract}
:
Remotely sensed data have huge importance to determine land use/cover changes for sustainable region planning and management. Variety of techniques in order to detect land cover dynamics using remote sensing imagery have been developed, tested and assessed with the results varying according to the change scenario, the information required and the imagery applied. In this study, the modified Change Vector Analysis (mCVA) technique was implemented on SPOT 4 and SPOT 5 multispectral (MS) data to monitor the dynamics of land use/land cover (LULC) change in Terkos Water Basin, İstanbul. mCVA was applied to multi-temporal data to compare the differences in the time-trajectory of the Tasseled Cap (TC) brightness, greenness and wetness for two successive time periods - 2003 and 2007. Gram Schmidt Orthogonalization Technique was used to derive the related TC coefficients for SPOT data. The efficiency of the technique was assessed based on error matrix. The overall accuracy and Kappa statistic was $84.32 \%$ and 0.81 , respectively. The results indicated that it is possible to produce accurate change detection maps with the help of mCVA and SPOT 4 \&SPOT 5 satellite data.
\end{abstract}

\section{INTRODUCTION}

Land use and land cover (LULC) change is an essential issue in monitoring and managing Earth surface. Due to the anthropogenic and natural activities, the Earth' surface is being significantly altered. These alterations are linked to other forms of environmental degradations and transformations such as habitat and biodiversity loss, invasive species and ground water depletion (Lambin and Strahler, 1994; Flores and Yool, 2007).

Accurate and efficient techniques are necessary to provide upto-date LULC change information. During recent decades, remotely sensed imagery became a major data source for LULC change detection because of the advantages of temporal data, synoptic view, fast data acquisition and digital format suitable for computer processing ( $\mathrm{Lu}$ et al., 2004). Many different remote sensing change detection algorithms are applied to determine LULC change detection and to produce thematic maps for monitoring, planning, and management of natural resources (Balik Sanli et al., 2007; Bektas Balcik, 2010). Coppin et al., (2004) give a comprehensive review of the wide range of change detection techniques.

In this study, Change Vector Analysis (CVA) was selected to exploit the full range of spectral information contained in the remotely sensed data to determine the magnitude and direction of change using SPOT 4 and SPOT 5 MS data from the years of 2003, and 2007. Change magnitude and direction were determined by using brightness, greenness and wetness axes of the Tasseled Cap Transform (Kauth-Thomas transform). LULC change dynamics were delineated by applying ISODATA classification. The performance of mCVA method was analyzed by calculating overall accuracy and Kappa statistic.

\section{STUDY AREA}

In this study, Terkos Water Basin, İstanbul was selected as study area. Lake Terkos is one of the most important drinking water sources of the Istanbul. The natural freshwater lake is located northwest Istanbul and is separated from the Black Sea with a thin land barrier (Figure 1). It lies between latitudes $40^{\circ} 19^{\prime} \mathrm{N}$ and $41^{\circ} 42^{\prime} \mathrm{N}$, and longitudes $28^{\circ} 29^{\prime} \mathrm{E}$ and $28^{\circ} 32^{\prime} \mathrm{E}$. The area of the basin is $632 \mathrm{~km}^{2}$ and the area of the Lake Terkos is $42 \mathrm{~km}^{2}$. The region includes agricultural areas, vegetated lands, a fresh water lake, mixed forest, wetlands and residential areas. Lake Terkos is an important vegetation area (Özhatay et al., 2003 ) and it provides approximately $25 \%$ of drinking water demand of Istanbul (Coşkun et al., 2006).

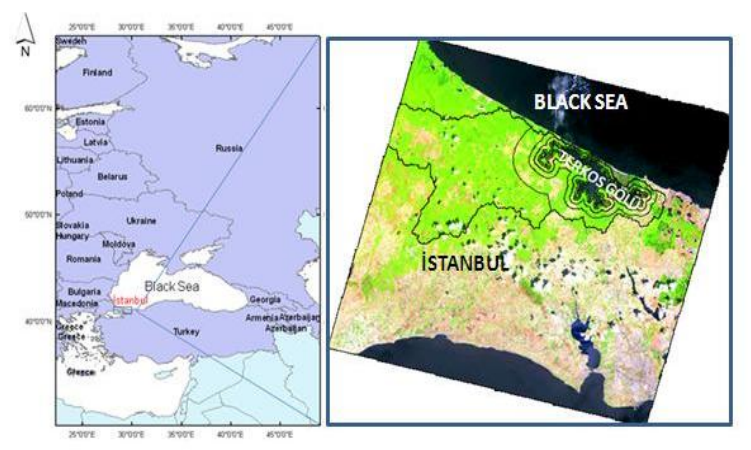

Figure 1. Location of İstanbul and Terkos Water Basin

Rapid industrialization, urbanization and population increase have caused to changes in landscape characteristics of the Istanbul. This urban growth causes dramatic problems in use of the natural resources such as fresh water supplies. 


\section{DATA AND METHODOLOGY}

\subsection{Data}

In this study, SPOT 4 XS (20 m) image acquired on July 24 , 2003 and SPOT 5 MS (10 m) acquired on August 14, 2007 were used (SPOT Image Copyright 2007, CNES). 1/5000 scaled aerial photographs and SPOT 5 Pan-sharpen $(2.5 \mathrm{~m})$ data were used for ground truth and accuracy assessment.

\subsection{Pre-processing}

The aim of remotely sensed data change detection is to compare the differences in the spatial representation of reflectance values of two points in time (Green et al., 1994; Yool and Flores, 2007). The preliminary requisite for accurate change detection is precise geometric and atmospheric correction of the multitemporal images. In the study, all images were geometrically corrected using first order polynomials and Ground Control Points (GCPs) collected from rectified SPOT 5 PAN data and 1/5000 scaled maps to eliminate geometric distortions, and to define images in a common Universal Transverse Mercator (UTM) coordinate system. The digital numbers of the images were transformed to radiance values according to the reference information from image header file. Radiance values were converted to at-satellite reflectance based on the method presented by Vermote et al., 1997. Dark Object Subtraction (DOS) atmospheric correction method applied to multi temporal images to remove haze caused by atmospheric phenomena (Chavez, 1988).

\subsection{Change Vector Analysis}

Change vector analysis (CVA) is a spectral differencing technique, the primary utility of which is the detection of all changes present in the input multispectral data (Malila, 1980). The method was first used to characterize magnitude and direction variations of a vector in an $\mathrm{n}$-dimensional spectral space defined by the axes of bands, transforms, or spectral features from a multi-temporal dataset (Malila, 1980). It is flexible enough to be effective when using diverse types of sensor data and radiometric change applications. CVA avoids spatial-spectral errors associated with post-classification comparison (Chen et al., 2003).

\subsection{Description of Tasselled Cap Transformation Features}

Brightness, greenness and wetness are the components of Tasseled Cap Transformation and descriptions of the components are given in Table 1 (Chris and Kauth, 1986; Lillesand and Kiefer, 1987). These components were used in mCVA to determine the dynamics of LULC changes that occurred in the selected region.

\begin{tabular}{|l|l|}
\hline Component & Description \\
\hline Brightness & $\begin{array}{l}\text { It is weighted sum of all bands, aligned in the } \\
\text { direction of the principal variation in soil } \\
\text { reflectance. }\end{array}$ \\
Wreenness & $\begin{array}{l}\text { It represents the contrast between the near- } \\
\text { infrared and visible bands (orthogonal to } \\
\text { brightness) } \\
\text { It relates to canopy and soil moisture } \\
\text { (orthogonal to brightness and greenness) }\end{array}$ \\
\hline
\end{tabular}

Table 1. Description of TC features

\subsection{Gram Schmidt Transformation and Tasselled Cap Parameters (Kauth -Thomas) Extraction}

Gram Schmidt Transformation (GST) is a mathematical tool that performs a rotational transformation in $\mathrm{n}$-dimensional space, resulting in a series of orthogonal axes, each of which is a linear combination of the original axes. Three Tasseled Cap parameters are named Brightness, Greenness and Wetness were extracted from SPOT 5 MS data by using GST based on Ivits et al., 2008. Samples of bare soil, green vegetation, and water were selected to derive the TC coefficients.

\subsection{Change Vector Analysis Based on TC Features}

After image pre-processing, CVA was applied using derived TC coefficients to reveal the dynamics of land use/land cover (LULC) changes in Terkos Water Basin, İstanbul. The TC coefficients derived for SPOT 5 (Bektaş Balçık, 2010) (Table 2) were used in a linear combination to transform 2003 dated SPOT 4 and 2007 dated SPOT 5 images into brightness, greenness and wetness features.

\begin{tabular}{|l|l|l|l|l|}
\hline SPOT 5 & Green & Red & $\begin{array}{l}\text { Near } \\
\text { Infrared }\end{array}$ & $\begin{array}{l}\text { Short Wave } \\
\text { Infrared }\end{array}$ \\
\hline Brightness & 0.201 & 0.397 & 0.548 & 0.707 \\
Greenness & -0.180 & -0.330 & 0.832 & -0.408 \\
Wetness & 0.388 & 0.573 & 0.013 & -0.724 \\
\hline
\end{tabular}

Table 2. TC coefficients for SPOT 5 data at satellite reflectance

In this study, an extended CVA experiment was implemented based on the spherical geometry approach introduced by (Allen and Kupfer, 2000) to measure vector longitude and colatitude. This method adds a new colatitude measurement to improve the change detection capability. A model in Erdas Imagine Modeler tool was created to calculate the results of extended CVA. The detailed description of the model is given in Figure 2 and the model is produced based on Flores and Yool, 2007.

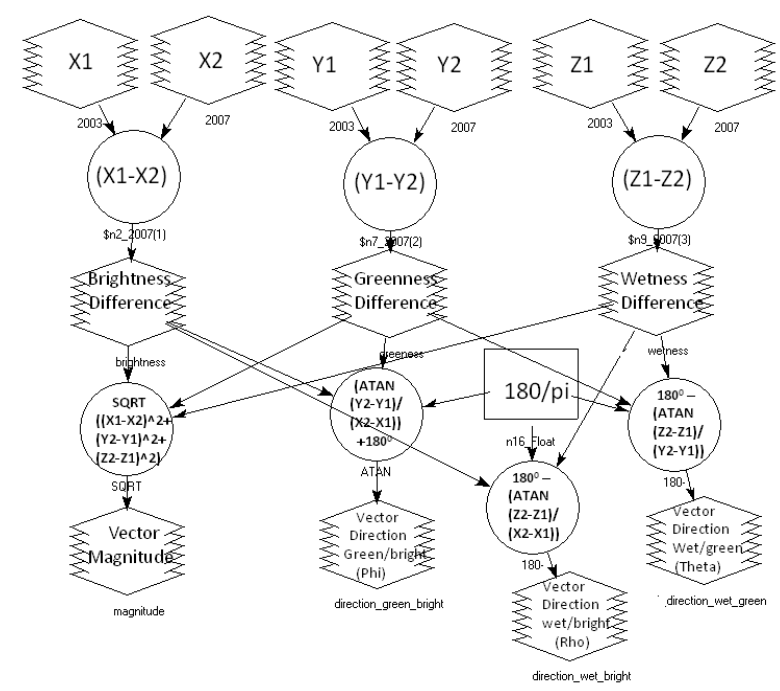

Figure 2. Graphical model script of the extended change vector analysis algorithm for SPOT 4 XS (2003) and SPOT 5 XS (2007) data.

This method derives the information about both the amounts and types of changes in the data. The output of the model includes the magnitude and three direction images. 
In order to determine changed and non-changed regions threshold value was determined using the remote sensing analyst's expert knowledge of the study area. Therefore, the histogram of change magnitude image is analyzed to detect change or no change threshold. Furthermore, Normalized Vegetation Index (NDVI) images of SPOT 4 and SPOT 5 were used for threshold determination.

\section{RESULTS}

In this study mCVA method was applied to different dated SPOT 4 and SPOT 5 MS data set by using derived TC coefficients. By using the derived coefficients brightness, greenness and wetness images were produced. The model produced several image maps that characterize land cover change in the Terkos Water Basin, İstanbul. Three TC 'difference' images; one change vector magnitude image, three vector direction images; and one final landscape dynamic image based on the derived brightness, greenness, and wetness were produced.

Magnitude image is given in Figure 3 categorized as changed, and no changed area. White colors showed the changed area and black colors showed the non-changed areas. Gray tones can be described as minimum, lower, medium and higher changes.

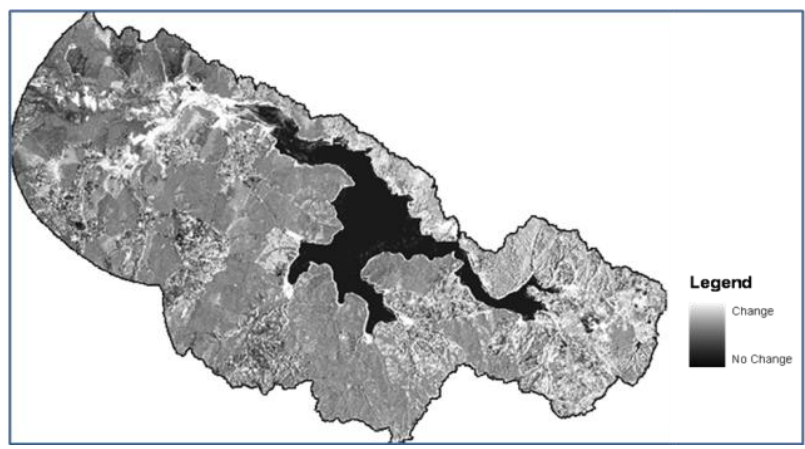

Figure 3. Change vector magnitude representing the intensity of change between a pixel's brightness, greenness, and wetness in 2003 and 2007.

This image represent the intensity of the change, which increases in the sand, agricultural areas, open spaces with little/or no vegetation and a part of vegetated areas. The largest increase in change vector magnitude is registered in agricultural fields because of the shape differences and heterogeneous product type in the area. The presence of growing crops and growing young trees at the time of SPOT 5 image acquisition resulted in extreme land cover changes in the area. The area that shows little change is urban areas and some of the agricultural fields. This area is under conservation by the low and urbanization is forbidden in the conservation zones. Because of this reason, urbanization is very limited in the area.

Magnitude image classified as changed and non-changed area based on threshold value. This value is determined by using statistic of the magnitude image and analyst knowledge about the study region. NDVI image helped for decision of the threshold value. The resultant image depicted in Figure 4. As a result of this process the area of changed and unchanged was found 3853 ha and 24884.84, respectively.

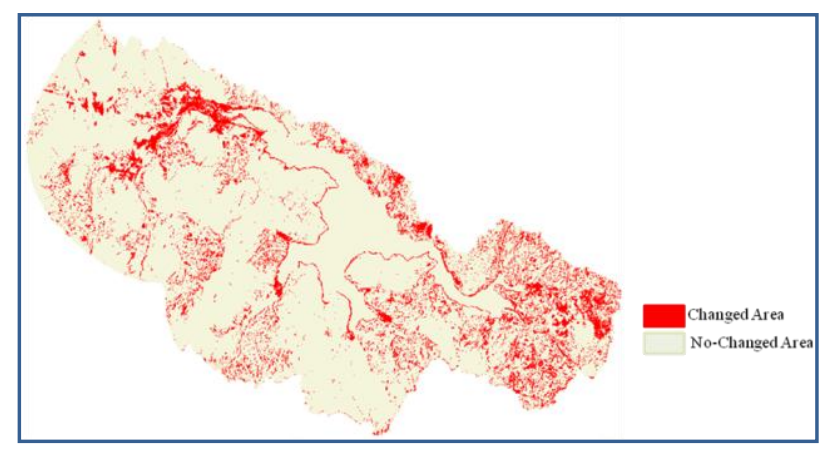

Figure 4. Changed and no-changed area

Direction images were produced based on given equations in Figure 2. Direction images for greenness/brightness, wetness/brightness and wetness/greenness were given in Figure 5 .

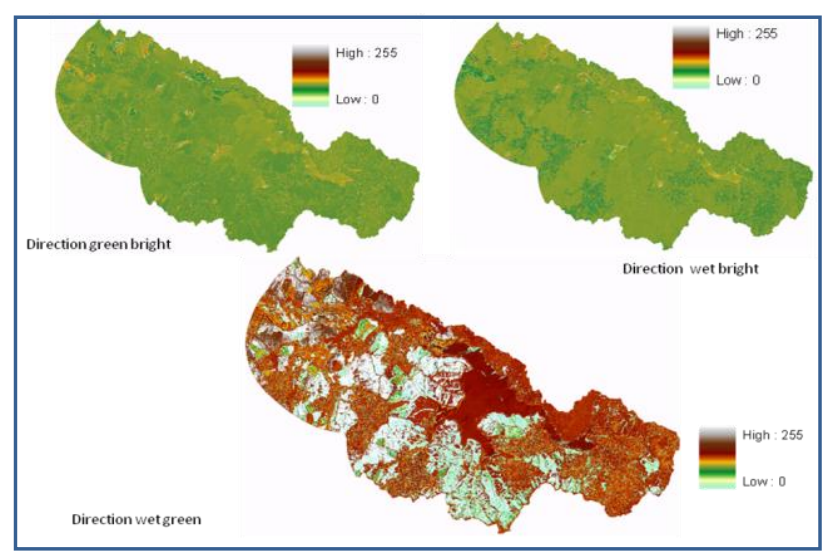

Figure 5. Direction images produced by mCVA

Longitude vector direction (direction green/bright) image shows a large brightness increase in the vegetated areas. Barren lands show increase in brightness. Conversely, most of the semi natural and mixed forest communities register a vector direction change towards the greenness axis. The colatitude vector direction (direction wet/green) image shows very small vector changes in the greenness-wetness plane. We can see very small changes in the open spaces with little or no vegetation. Also, it is possible to see some changes in the sand dune of the study area. The colatitude vector direction is the vector direction component that shows larger variation of vector displacements. The results were quite similar from the longitude and colatitude vector direction image. Accounting for vector changes in the wetness-brightness plane, colatitude was able to enhance more bright features in the vegetated and agricultural lands. The most important disagreement of colatitude with the other two vector direction components is at the areas occupied with young generation trees in the north site of the lake (sandy area).

At the last stage, three direction images were stacked and unchanged areas were masked from the direction images before ISODATA unsupervised classification. As a result of the classification six categories were produced such as high greenness, high brightness, high wetness, medium greenness, medium brightness and medium wetness. The most dynamic landscapes were considered in this study to avoid overestimating because of small changes. Total area of change for each category obtained by classifying direction and magnitude images is displayed in Figure 6 and Table 3. 


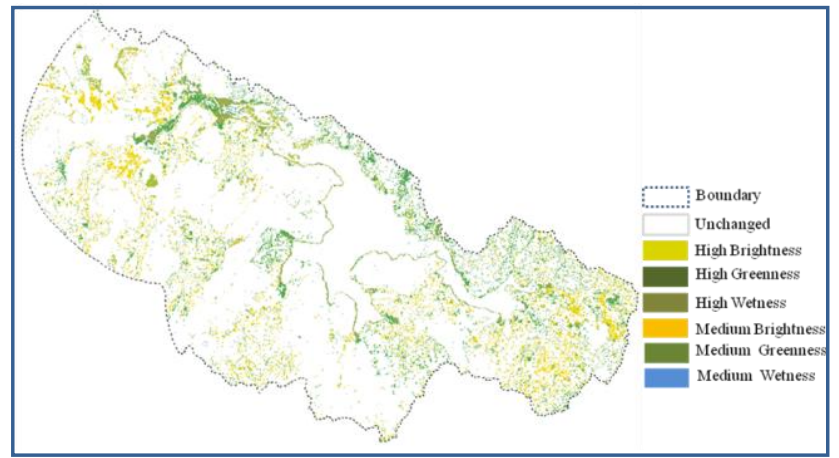

Figure 6. ISODATA classification image

In Figure 6 each class indicates intensity of vector change in any direction within the Tasseled Cap Transformation.

\begin{tabular}{|l|l|l|l|}
\hline Class & Value & Area (ha) & $\%$ \\
\hline 0 & No change & 24716.44 & 86.51 \\
1 & High wetness & 350.12 & 1.22 \\
2 & High greenness & 650.88 & 2.28 \\
3 & High brightness & 1282.20 & 4.49 \\
4 & Medium wetness & 131.20 & 0.46 \\
5 & Medium greenness & 1296.60 & 4.54 \\
6 & Medium brightness & 142.56 & 0.50 \\
\hline
\end{tabular}

Table 3. Landscape dynamic classes and their corresponding area in the Terkos

After image classification, accuracy of the resultant image was assessed. Accuracy assessment is very important for understanding the detected change results and employing these results for land management, urban land planning and decisionmaking (Foody, 2002). In this study, overall accuracy and Kappa analysis were used to perform classification accuracy assessment based on error matrix. Overall accuracy and Kappa statistics were calculated as $84.32 \%$ and 0.81 , respectively.

\section{CONCLUSIONS}

In this study, mCVA used to detect the most dynamic areas in Terkos Water Basin, İstanbul. Three TC features (brightness, greenness and wetness) were derived for SPOT 5 MS data to produce three difference, one magnitude and three direction images using 2003 dated SPOT 4 and 2007 dated SPOT 5 MS data. Classified vector magnitude and direction components were used for the selection of dynamic landscapes. The advantage of the mCVA is it has the ability to process any number of spectral bands but it is difficult to decide changed classes and threshold value. The sensitivity of the CVA was enhanced by using polar coordinates to represent vector directions. CVA and extended polar coordinates improved the ability to determine landscape dynamics in this heterogeneous environment.

\section{ACKNOWLEDGMENT}

The authors would like to thank the SPOT OASIS project for providing the 2007 dated SPOT 5 MS and PAN images.

\section{REFERENCES}

Balik Sanli F., Bektaş Balçık F., and Goksel C., 2007. Defining temporal spatial patterns of mega city Istanbul to see the impacts of increasing population. Environmental Monitoring and Assessment, 146, 1-3, pp. 267-275.

Bektaş Balçık, F., 2010. Mapping and Monitoring Wetland Environment by Analysis of Different Satellite Images and Field Spectroscopy'. Istanbul Technical University, $\mathrm{PhD}$ thesis.

Chavez, P. S., 1988. An improved dark-object subtraction technique for atmospheric scattering correction of multispectral data. Remote Sensing of Environment, 24, pp. 459-479.

Chen, J., Gong, P., He, C. and Shi, P., 2003. Land-use/landcover change detection using improved change-vector analysis. Photogrammetric Engineering and Remote Sensing, 69, pp. 379.

Coppin, P., Jonckheere, I., Nackaerts, K., Muys, B., Lambin, E., 2004. Digital change detection methods in ecosystem monitoring; a review. International Journal of Remote Sensing, 25, pp. 1565-1596.

Coskun, H. G., Gulergun, O., Yilmaz, L., 2006. Monitoring of protected bands of Terkos drinking water reservoir of metropolitan 'Istanbul near the Black Sea coast using satellite data. International Journal of Applied Earth Observation and Geoinformation, 8, pp. 49-60.

Crist, E.P., and Kauth, R. J., 1986. The Tasseled Cap demystified. Photogrammetric Engineering and Remote Sensing, 52, pp. 81-86.

Flores, S. E., Yool, S. R., 2007. Sensitivity of change vector analysis to land cover change in an arid ecosystem. International Journal of Remote Sensing, 28, pp. 1069-1088.

Foody, G. M., 2002: Status of land cover classification accuracy assessment. Remote Sensing of Environment. Vol. 80, pp. 185201.

Green, K., Kempka, D. and Lackey, L., 1994. Using remote sensing to detect and monitor land-cover and land-use change. Photogrammetric Engineering and Remote Sensing, 60, pp. 331-337.

Ivits, E., Lamb A., Langar, F., Hemphill, S., Koch, B., 2008. Orthogonal transformation of segmented SPOT 5 images: Seasonal and geographical dependence of the tasseled cap parameters. Photogrammetric Engineering \& Remote Sensing, 74, 11, pp. 1351-1364

Lambin, E.F., and Strahler, A. H., 1994. Change vector analysis in multi- temporal space: A tool to detect and categorize landcover change processes using high temporal-resolution satellite data. Remote Sensing of Environment, 48:231-244.

Lillesand, T. M., and Kiefer, R. W., 1987. Remote Sensing and Image Interpretation (New York: John Wiley \& Sons).

Lu, D., Mausel, P., Brondizio, E., and Moran, E., 2004. Change detection techniques. International Journal of Remote Sensing, 25 , pp. 2365-2407.

Malila, W.A., 1980. Change vector analysis: an approach for detecting forest changes with Landsat. Proceedings of the Sixth Annual Symposium Machine Processing of Remotely Sensed Data, Purdue University, West Lafayette, IN, pp. 326-335. 
International Archives of the Photogrammetry, Remote Sensing and Spatial Information Sciences, Volume XXXIX-B7, 2012 XXII ISPRS Congress, 25 August - 01 September 2012, Melbourne, Australia

Ozhatay, N. B. A., Atay, S., 2003. Important Plant Areas of Turkey. WWF Turkiye, MAS Press (in Turkish).

Vermote, E. F., Tanre, D., Deuze, J. L., Herman, M., Morcrette, J. J., 1997. Second simulation of the satellite signal in the solar spectrum: An overview. IEEE Transactions on Geoscience and Remote Sensing, 35, pp. 675-686. 